\title{
Expression levels and clinical significance of hepsin and HMGB1 proteins in cervical carcinoma
}

\author{
HUI CHENG ${ }^{*}$, WEIQI WANG ${ }^{*}$, YANLING ZHANG, BEI ZHANG, JIE CHENG, PENG TENG and XIN TANG \\ Department of Obstetrics and Gynecology, Xuzhou Central Hospital, Xuzhou, Jiangsu 221009, P.R. China
}

Received August 5, 2016; Accepted March 24, 2017

DOI: 10.3892/ol.2017.6116

\begin{abstract}
This study assessed the hypothesis that the protein levels of high mobility group box 1 (HMGB1) and hepsin can be used as markers for diagnosis and prognosis in cervical carcinoma. Seventy patients with cervical cancer who were hospitalized in Xuzhou Central Hospital from May 2008 to June 2010 and underwent surgical treatment were selected for the observation group. At the same time, 20 patients with cervical benign lesions who underwent tumor stripping or accessory resection were selected for the control group. Immunohistochemical (SP) detection methods were used to detect hepsin and HMGB1 protein levels in tissues. The positive rates of HMGB1 cells in normal, paracancerous and cancerous cervical tissues were $5.0 \%(1 / 20), 22.9 \%(16 / 70)$ and $95.7 \%(67 / 70)(\mathrm{F}=24.581, \mathrm{P}=0.001)$ respectively. The positive rates of hepsin in normal, paracancerous and cancerous cervical tissues were respectively $10 \%(2 / 20), 61 \%(43 / 70)$ and $90 \%(63 / 70)(\mathrm{F}=11.538, \mathrm{P}=0.001)$. The HMGB1 expression level was related to the degree of tumor differentiation, lymph node metastasis and TNM stage $(\mathrm{P}<0.05)$. While the level of hepsin expression was related to the degree of tumor differentiation, invasion depth, lymph node metastasis and TNM stage $(\mathrm{P}<0.05)$. Furthermore, a positive correlation between the levels of hepsin and HMGB1 was found ( $\mathrm{r}=15.27$, $\mathrm{P}<0.05)$. The overall 5-year survival rates of patients with high expression of HMGB1 (+++) and low expression of HMGB1 $(+$ to ++$)$ were respectively 51.2 and $29.2 \%(\mathrm{HR}=11.637$, 95\% $\mathrm{CI}=4.351-38.213 ; \mathrm{P}=0.002)$. The overall 5 -year survival rates of patients with high hepsin expression $(+++)$ and low hepsin expression (+ to ++ ) were respectively 41.3 and $35.3 \%$ $(\mathrm{HR}=10.143,95 \% \mathrm{CI}=4.285-33.275 ; \mathrm{P}=0.006)$. Based on our results, the higher the levels of expression of hepsin and HMGB1 in tissues the higher the degree of invasiveness of the cervical cancers, and the worse the prognoses for the patient.
\end{abstract}

Correspondence to: Dr Yanling Zhang, Department of Obstetrics and Gynecology, Xuzhou Central Hospital, 199 Jiefang South Road, Xuzhou, Jiangsu 221009, P.R. China

Email: q376054@163.com

Key words: cervical cancer, high mobility group box 1 protein, hepsin, invasion

\section{Introduction}

Cervical cancer is the most common malignant tumor of the female reproductive system in China. It is diagnosed mostly at advanced stages when its prognosis is poor. The 5 -year survival rate is only $60 \%$. The survival rate of patients with distant metastasis is even lower. In recent years, with the progresses made in surgical treatments, radiotherapy and chemotherapy, the 5-year survival rate of patients has improved. However, the long-term survival rate is still not satisfactory. Therefore, newer approaches like gene therapy against tumor proliferation and invasion have gained widespread interest (1). Hepsin is a recently discovered gene, the hepsin protein is involved in the dissolution of the cell membrane matrix and results in abnormal signal transduction between cells, moreover, the high expression of hepsin is related to the development and metastasis of malignant tumors (2). The high mobility group box 1 (HMGB1) protein, belonging to high mobility group protein family, is a non-histone chromosomal protein, expressed in the early stages of embryonic development, and is almost absent from highly differentiated tissues. HMGB1 has been confirmed to be present in malignant, invasive and poor-prognosis tumors (3). In this study, the immunohistochemical SP method was used to detect HMGB1 and hepsin protein expression in cervical carcinoma and control tissues. We studied the relationship of the levels of expression of these proteins in tumors with their rates of invasion, and metastasis, as well as their prognoses. Our results point to possible pathogenesis mechanisms, aids the search for new tumor markers to more accurately predict prognosis, and provide new ideas for genetic target treatments of cervical cancer.

\section{Patients and methods}

Patient data. Seventy cervical cancer patients who initially received surgical treatments and had completely preserved clinical and pathological data, in the Department of Obstetrics and Gynecology, Xuzhou Central Hospital from May 2008 to June 2010, were selected to participate in the study. Inclusion criteria included: i) A definite pathological diagnosis; ii) clinical data were complete; iii) no radiotherapy or chemotherapy had been performed before operation; and iv) there were no brain, liver, kidney or other organ dysfunctions. The age of the patients ranged from 22 to 74 years with an average of $42.6 \pm 13.2$ years. There were 59 cases of squamous cell carcinoma and 11 cases of adenocarcinoma. Classification 

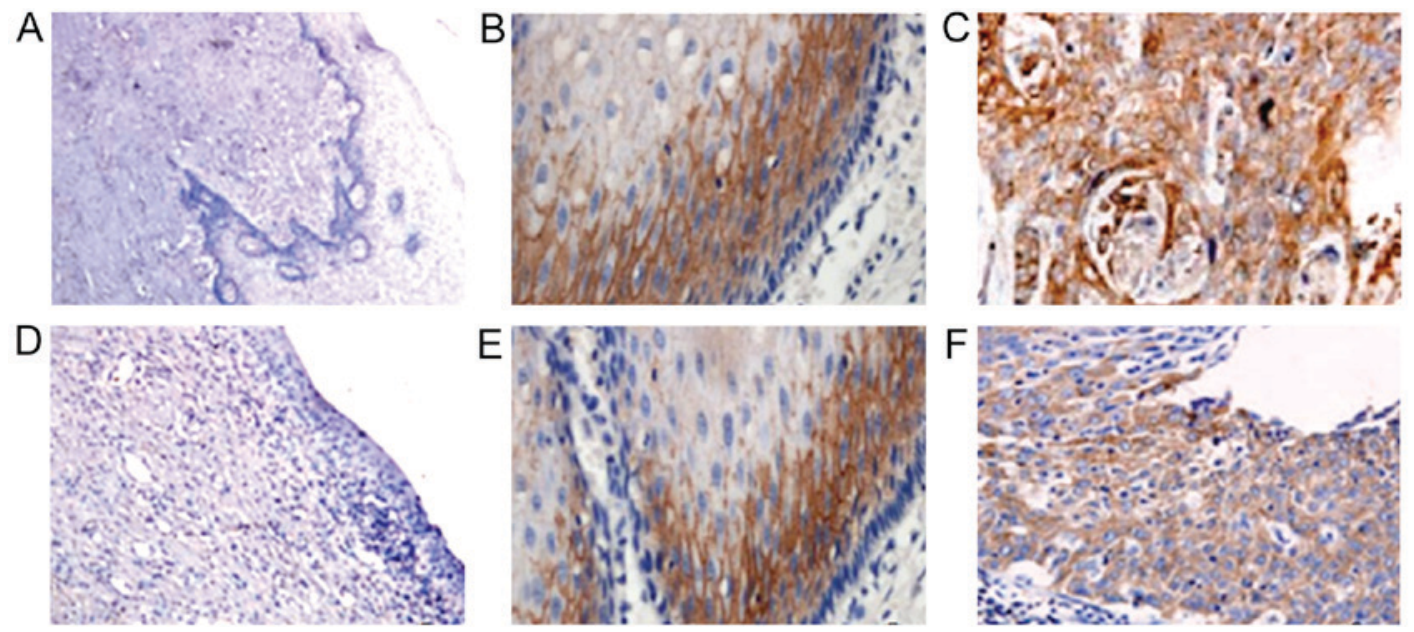

Figure 1. Expression of HMGB1 and hepsin in normal cervical tissues, paracancerous tissues and cervical cancer tissues. (A) HMGB1 protein expression was negative in normal cervical tissues. (B) HMGB1 protein expression in paracancerous tissues was pale yellow moderately positive (++). (C) HMGB1 protein expression in cervical carcinoma tissues was tan color strong positive (+++), and located in the cytoplasm. (D) Hepsin protein expression in normal cervix group was negative. (E) Hepsin protein expression was yellow positive (++) in paracancerous tissues. (F) Hepsin protein expressed in cervical carcinoma tissues was tan strong positive (+++), and located in the cytoplasm. HMGB1, high mobility group box 1.

according to the FIGO standards (4) placed 28 cases in the low degree (I and II classification), and 42 in the high degree (III and IV classification). Also, based on pathological differentiation, there were 8 patients with high differentiation, 25 with moderate differentiation, and 37 with poor differentiation, and 47 patients had lymph node metastasis, and $23 \mathrm{did}$ not have any. Tissues $2 \mathrm{~cm}$ away from the tumor border were used for the paracancerous tissue group, and 20 cases of normal cervical tissue, from accessory resections were taken for the control group. Comparing the average ages of the control and the cervical cancer groups, the difference was not statistically significant $(\mathrm{P}>0.05)$, indicating that the division in both groups could be used for other comparisons. All subjects signed written informed consents and the Ethics Committee of Xuzhou Central Hospital approved the study. The 70 cervical cancer patients were followed-up; the last day of the follow-up was July 1, 2015, and the average follow-up time was 52.1 months.

Immunohistochemistry. Paraffin-embedded tissues were collected and immunohistochemistry used the SP method. Mouse polyclonal hepsin antibody (dilution, 1:100; cat. no. sc-517056) and mouse monoclonal HMGB1 antibody (dilution, 1:150; cat. no. sc-135809) (both from Santa Cruz Biotechnology, Inc., Santa Cruz, CA, USA) were used. SP immunohistochemical kits were purchased from Beijing Zhongshan Golden Bridge Biotechnology Co. (Beijing, China). Experimental steps were conducted according to the kit's specifications. Phosphatase-buffered saline (PBS) was used instead of primary antibody as a negative control, and the positive control was an antibody known to be positive on the samples.

Result judgments. HMGB1 positive staining signal was brown and disseminated in small particles, all of which were located in the cytoplasm of the cells. The hepsin protein was distributed in the nucleus and the cytoplasm and consisted of brownish yellow or brown particles. For each slice, 10 consecutive views
Table I. Expression levels of HMGB1 and hepsin in different tissues (n, \%).

\begin{tabular}{lccc}
\hline & & \multicolumn{2}{c}{ Positive rate } \\
\cline { 3 - 4 } Tissues & Cases & HMGB1 & Hepsin \\
\hline Normal & 20 & $5.0 \%(1 / 20)$ & $10 \%(2 / 20)$ \\
Paracancerous & 70 & $22.9 \%(16 / 70)$ & $61 \%(43 / 70)$ \\
Cervical cancer & 70 & $95.7 \%(67 / 70)$ & $90 \%(63 / 70)$ \\
F-value & & 24.581 & 11.538 \\
P-value & & 0.001 & 0.001 \\
\hline
\end{tabular}

HMGB1, high mobility group box 1 .

at x400 magnification were examined, 200 cells were counted in each view, and all the positive cells were counted, in order to calculate the positivity rate. The percentage of positive cells were then calculated, and each sample was assigned points: $0-5 \%$ was noted as 0 points, $6-25 \%$ was noted as 1 point, $26-50 \%$ was 2 points, and more than $50 \%$ was noted as 3 points. Additionally, the staining intensity was also ranked by points: Unpigmented cells were marked 0 points, pale yellow cells were given 1 point, brownish-yellow cells were given 2 points, and brown cells were given 3 points. Multiplying the points of both scores resulted in definite comprehensive scores: A score of 0-1 meant tissue was the negative for HMGB1 or hepsin staining, a score of 2-3 meant the tissue was weakly positive (+), 4-6 meant tissue was moderately positive (++) and a score of 9 meant the tissue was strongly positive $(+++)$.

Statistical analysis. The SPSS software package (SPSS, Inc., Chicago, IL, USA) was used for data analysis, measurement data were expressed by mean $\pm \mathrm{SD}$. The t-test (Fisher's exact test) was used in comparison between groups, $\chi^{2}$ test and exact tests were used to compare the rate of two samples, and for correlations the Pearson's test was adopted. The survival rate 
Table II. Relationship of HMGB1 and hepsin with clinicopathological factors of cervical cancer.

\begin{tabular}{|c|c|c|c|c|c|}
\hline \multirow[b]{2}{*}{ Pathological factors } & \multirow[b]{2}{*}{ Cases } & \multicolumn{2}{|c|}{ HMGB1 } & \multicolumn{2}{|c|}{ Hepsin } \\
\hline & & + & P-value & + & P-value \\
\hline \multicolumn{6}{|l|}{ Age (years) } \\
\hline$<55$ & 48 & 47 (97.9) & \multirow[t]{2}{*}{0.257} & $44(91.7)$ & \multirow[t]{2}{*}{0.126} \\
\hline$\geq 55$ & 22 & $20(90.9)$ & & $18(81.8)$ & \\
\hline \multicolumn{6}{|l|}{ Pathological types } \\
\hline Localization & 34 & $31(91.2)$ & \multirow[t]{2}{*}{0.204} & $28(82.4)$ & \multirow[t]{2}{*}{0.067} \\
\hline Infiltrating & 36 & $36(100)$ & & $35(97.2)$ & \\
\hline \multicolumn{6}{|c|}{ Degree of differentiation } \\
\hline Poor & 37 & $36(97.3)$ & \multirow[t]{2}{*}{0.191} & $36(97.3)$ & \multirow[t]{2}{*}{0.044} \\
\hline High and middle & 33 & $30(90.9)$ & & $27(81.8)$ & \\
\hline \multicolumn{6}{|l|}{ Depth of invasion } \\
\hline $\mathrm{T} 1+\mathrm{T} 2$ & 14 & $11(78.6)$ & \multirow[t]{2}{*}{0.043} & $8(57.1)$ & \multirow[t]{2}{*}{0.013} \\
\hline $\mathrm{T} 3+\mathrm{T} 4$ & 56 & $56(100)$ & & $55(98.2)$ & \\
\hline \multicolumn{6}{|c|}{ Lymph node metastasis } \\
\hline Negative & 23 & $20(87.0)$ & \multirow[t]{2}{*}{0.048} & $18(78.3)$ & \multirow[t]{2}{*}{0.035} \\
\hline Positive & 47 & $47(100)$ & & $45(95.7)$ & \\
\hline \multicolumn{6}{|l|}{ TNM stage } \\
\hline $\mathrm{I}+\mathrm{II}$ & 28 & $25(89.3)$ & \multirow[t]{2}{*}{0.087} & $24(85.7)$ & \multirow[t]{2}{*}{0.021} \\
\hline III+IV & 42 & $42(100)$ & & 39 (92.9) & \\
\hline
\end{tabular}

HMGB1, high mobility group box 1 .

was calculated using the Kaplan-Meier survival curve and was analyzed by the log-rank test. A $\mathrm{P}<0.05$ was considered to indicate a statistically significant difference.

\section{Results}

The expression of HMGB1 and hepsin in cervical carcinoma, paracancerous and normal tissues. HMGB1 was mainly expressed in the cytoplasm of cervical cancer cells, and was almost absent from normal tissues (only 5\% of them had some expression). The positive rate for paracancerous tissues was $22.9 \%(16 / 70)$, and the differences between these two groups had statistical significance $(\mathrm{P}<0.05)$. The positive expression rate of HMGB1 in cervical cancer tissues was $95.7 \%$ (67/70), and when compared to the rates in the normal and paracancerous groups the differences were statistically significant $(\mathrm{P}<0.05)$.

The hepsin protein was mainly expressed in the cytoplasm of the cervical cancer cells, and there were only 2 cases of hepsin expression in 20 cases of normal tissues (positive rate of $10 \%$ ). The positive expression rate in paracancerous tissues was $61 \%(43 / 70)$, and in the cervical carcinoma group was $90 \%$ (63/70), the differences among the three groups were statistically significant $(\mathrm{P}<0.05)$ (Table I and Fig. 1).

Relationship between expression of HMGB1 and hepsin with the pathological and clinical signs of cervical cancer. In 70 cases of cervical cancer, the expression of HMGB1 was independent of age, histological type, the degree of differentiation, and TNM stage (P-values $>0.05$ ). Only the depth of tumor
Table III. Correlation analysis of hepsin and HMGB1 in cervical cancer.

\begin{tabular}{lrrrr}
\hline & \multicolumn{2}{c}{ HMGB1 } & & \\
\cline { 2 - 3 } Hepsin & + & - & R-value & P-value \\
\hline+ & 47 & 7 & & \\
- & 1 & 15 & 15.27 & 0.01 \\
\hline
\end{tabular}

HMGB1, high mobility group box 1 .

invasion, and presence of lymph node metastasis correlated with the expression of HMGB1 $(\mathrm{P}<0.05)$.

The expression of hepsin was not related to the tumor pathological type $(\mathrm{P}>0.05)$. But, it was related to the degree of differentiation, the depth of invasion, the presence of lymph node metastasis and the TNM stage (P-values <0.05) (Table II).

Correlation analysis of hepsin and HMGB1. In 70 cases of cervical cancer tissues, the expressions of hepsin and HMGB1 were simultaneously positive $67.1 \%$ (47/70), and simultaneously negative $21.4 \%(15 / 70)$, here was a positive correlation between them $(r=15.27, \mathrm{P}<0.05)$ (Table III).

Comparison of prognosis of cervical cancer patients with different expression intensity of hepsin and HMGB1 protein. All subjects were followed-up for 5 years. On July 1, 2015, 
Table IV. Survival analysis of cervical cancer patients with different expression intensity levels for hepsin and HMGB1 protein.

\begin{tabular}{|c|c|c|c|c|c|c|}
\hline Category & Cases & $\begin{array}{l}\text { 5-year survival } \\
\text { rate }(\%)\end{array}$ & $\begin{array}{l}\text { Median survival } \\
\text { time (month) }\end{array}$ & HR value & $95 \% \mathrm{CI}$ & P-value \\
\hline \multicolumn{7}{|c|}{ HMGB1 protein expression } \\
\hline+ to ++ & 43 & $22(51.2)$ & 54.1 & 11.637 & $4.351-38.213$ & 0.002 \\
\hline+++ & 24 & 7 (29.2) & 24.8 & & & \\
\hline \multicolumn{7}{|c|}{ Hepsin protein expression } \\
\hline+ to ++ & 46 & $19(41.3)$ & 41.6 & 10.142 & $4.285-33.275$ & 0.006 \\
\hline+++ & 17 & $6(35.3)$ & 27.3 & & & \\
\hline
\end{tabular}

HMGB1, high mobility group box 1; HR, hazard ratio; CI, confidence interval.

66 patients completed their follow-up, while 3 patients with hepsin positive expression and 1 with HMGB1 positive expression failed to complete it. The rate of failure to complete the 5-year follow-up was $5.7 \%$, and the data of patients without a complete follow-up were not used anymore. During the follow-up period, in the 66 cases of cervical cancer patients, $34(51.5 \%, 34 / 66)$ died, and $32(48.5 \%, 32 / 66)$ were alive, the 5-year survival rate was $39.3 \%$, and the median survival time was 36.9 months. The log-rank test showed that expression of hepsin and HMGB1 protein (either strong or weak) were related to the patients' 5-year survival rate and median survival time $(\mathrm{P}<0.05)$. Comparing the risk of death $(\mathrm{HR})$ in cervical cancer patients with the strongly positive expression of hepsin and HMGB1 protein with weakly and moderately positive expressions, the differences had statistical significance $(\mathrm{P}<0.05)($ Table IV).

\section{Discussion}

Cervical cancer is one of the most common malignant tumors in females. The incidence rate increases year by year, with a high degree of malignancy and a high mortality rate. The typical features of malignant tumors are the invasiveness and high metastasis rates. It is thought that cell regulatory factors and other patient inherent factors play an important role in allowing for invasion and metastasis of the tumors. A large number of clinical trials have shown that patients with high expression of invasive biological factors have a worse prognosis. Therefore, it is very important to find new gene therapy targets for cervical cancer $(1,2)$. Study has shown that hepsin and HMGB1 proteins are involved in regulating the development of malignant tumors (3). This study was conducted to investigate the expression of hepsin and HMGB1 proteins in cervical carcinoma tissues, and to explore the relationship between their expression and the tumor proliferation, differentiation, and metastasis. We aimed at providing a theoretical and experimental basis for early diagnosis and prognosis, and new insights for more targeted treatments.

The relationship between HMGB1 protein expression and cervical cancer has been studied. HMGB1 is a cytokine discovered 30 years ago, it is believed to be important for the survival, proliferation, invasion and metastasis of malignant tumor cells, but the research on it is still in the exploratory stages (4). In recent years, many studies have linked the expression of
HMGB1 to invasion, distant metastasis and poor prognosis in gastric, colorectal cancer and pancreatic cancers (5-9). However, study of HMGB1 in cervical cancer is scarce, and its pathogenic mechanisms are still not clear. A group of researchers detected the expression of HMGB1 in 3 kinds of cervical cancer cell lines (C-4I, CaSki and ME-180) (10), their results showed that HMGB1 was barely expressed in normal cervical cells, but overexpressed in a highly invasive cervical cancer cell line. Others found that the expression of HMGB1 in cervical cancer was higher than that in normal cervical tissues, and the positivity rate in invasive cervical cancer was significantly higher than that in the pre-invasive carcinoma tissues (11). Yet another study showed HMGB1 being highly expressed in recurrent squamous cell carcinoma of the cervix, compared to other tumor markers, the specificity of HMGB1 was higher, it was suggested that HMGB1 expression levels could be used as a tumor marker to evaluate the prognosis of patients with cervical squamous-cell carcinoma (12). In our study immunohistochemical methods were used to detect the expression of HMGB1 protein in cervical normal and cancer tissues, and results indicated that the HMGB1 protein was not expressed in normal cervical tissues, but highly expressed in cervical cancer tissues. Moreover, a higher positive expression rate of HMGB1 was found in cervical cancer patients with high tumor infiltrating degrees and lymph node metastases. Cervical cancer patients with positive expression of HMGB1 protein had a poor prognosis, which was consistent with findings of others and further suggests a link between HMGB1 overexpression and the development of cervical cancer.

At present, there is no unified theory on the role of HMGB1 in the development of cervical cancer. It was found that HMGB1 stimulates the endothelial progenitor cell migration and neovascularization using its receptor RAGE (13). Also, the expression of HMGB1 was correlated with the expression of the RAGE protein in cervical squamous cell carcinoma tissues, suggesting positive feedback signal is important for cervical cancer. In addition, it was shown that HMGB1 led to MAPKs phosphorylation by Ras and MAPK pathway, thus activating the nuclear factor $-\kappa \mathrm{B}(\mathrm{NF}-\kappa \mathrm{B})$, which in turn activated gene transcription to promote cell proliferation and differentiation, tumor formation and metastasis (14). Studies have shown that in the process of invasion and metastasis of cervical cancer cells, HMGB1 may be related to the activation of matrix metalloproteinases (MMPs) and protein 
hydrolases such as MMP-2 and MMP-9, thus dissolving the extracellular matrix and promoting cancer cell invasion and distant metastases (15). Nevertheless, further study is needed in order to clarify the specific mechanisms and pathways involved.

The hepsin gene was first found in the cDNA of hepatocellular carcinoma cells, and mainly locates on the human chromosome 19 (q11-q13.2) (16). The hepsin protein is a type II transmembrane serine protease. It is involved in the dissolution of the extracellular matrix and the abnormal signal transduction between cancerous cells. The dissolution of extracellular matrix is a prerequisite for invasion and metastasis of cancer cells, however, the mechanisms by which hepsin acts are not clear. It was found that hepsin could dissolve extracellular basement membrane and guide cancer cell invasion and metastasis through the activation of a pathway for the plasminogen activator (pro-uPA) of the precursor urokinase type (17). It has been proposed that hepsin may activate the double chain hepatocyte growth factor, which leads to the degradation of the cell basement membrane resulting in a weaker intercellular adhesion force, that facilitates cellular movement, invasion and metastasis (18). Others believe that the hepsin gene may act on cancer cells through the $\mathrm{HGF} / \mathrm{Met}$ pathway, reducing the adhesion between cells, and thereby promoting invasion and metastasis of malignant tumor cells (19).

A large number of studies have confirmed that hepsin protein is highly expressed in various tumor tissues (20-22). Hepsin is highly overexpressed in prostate cancer tissues, and its expression level correlates with the Gleason score, the prognosis and recurrence rate of the tumor (23). The expression of hepsin in endometrial carcinoma is significantly higher than that in the normal endometrium and in benign hyperplasia, and the levels of expression are related to pathological factors such as infiltration and metastasis (24). Therefore, hepsin is considered a group of key enzymes promoting tumor metastasis $(25,26)$. Then again, there are few studies on the clinical levels of hepsin gene in cervical cancer, although it may be a new marker of cervical cancer and a promising therapeutic target. In this study the expression of hepsin in different cervical tissues was examined. The results suggest that the expression rate of hepsin protein changes gradually start at a low level in normal cervical tissue, increasing in paracancerous tissue and then getting high in cervical cancer tissue. Importantly, the levels of hepsin were related to the differentiation degree, invasion depth, lymph node metastasis presence and TNM stage of cervical cancer, which suggests that hepsin is closely linked with the development, metastasis and invasion of cervical cancer. We conclude the levels of hepsin may be considered a risk factor for tumor development and metastasis.

In conclusion, this study showed that the expression levels of hepsin and HMGB1 were related to the depth of invasion, the presence of lymph node metastasis of cervical cancer and the prognosis for patients. This suggests that both proteins may play a synergistic role in the genesis and development of cervical cancer. We believe that the combined detection of hepsin and HMGB1 expression is helpful in the diagnosis and prognosis of cervical cancer, and propose them as a pair of new markers for cervical cancer. Moreover, blocking both signal transduction pathways may become a successful strategy for inhibiting cervical cancer.

\section{References}

1. Li J, Kang LN and Qiao YL: Review of the cervical cancerdisease burden in mainland China. Asian Pac J Cancer Prev 12: 1149-11453, 2011.

2. Tanimoto H, Yan Y, Clarke J, Korourian S, Shigemasa K, Parmley TH, Parham GP and O'Brien TJ: Hepsin, a cell surface serine protease identified in hepatoma cells, is overexpressed in ovarian cancer. Cancer Res 57: 2884-2887, 1997.

3. Ulloa L and Messmer D: High-mobility group box 1 (HMGB1) protein: friend and foe. Cytokine Growth Factor Rev 17: 189-201, 2006.

4. Kong TW, Piao X, Chang SJ, Paek J, Lee Y, Lee EJ and Ryu HS: A predictive model for parametrial invasion in patients with FIGO Stage IB cervical cancer: individualized approach for primary treatment. Int J Gynecol Cancer 26: 184-191, 2016.

5. Ohmori H, Luo Y and Kuniyasu H: Non-histone nuclear factor HMGB1 as a therapeutic target in colorectal cancer. Expert Opin Ther Targets 15: 183-193, 2011.

6. Chung HW, Lee SG, Kim H, Hong DJ, Chung JB, Stroncek D and Lim JB: Serum high mobility group box-1 (HMGB1) is closely associated with the clinical and pathologic features of gastric cancer. J Transl Med 7: 38, 2009.

7. Moriwaka Y, Luo Y, Ohmori H, Fujii K, Tatsumoto N, Sasahira T and Kuniyasu H: HMGB1 attenuates anti-metastatic defense of the lymph nodes in colorectal cancer. Pathobiology 77: 17-23, 2010.

8. Cebrián MJ, Bauden M, Andersson R, Holdenrieder S and Ansari D: Paradoxical role of HMGB1 in pancreatic cancer: tumor suppressor or tumor promoter? Anticancer Res 36: 4381-4389, 2016

9. Liu Z, Du R, Long J, Guo K, Ge C, Bi S and Xu Y: microRNA-218 promotes gemcitabine sensitivity in human pancreatic cancer cells by regulating HMGB1 expression. Chin J Cancer Res 27: 267-278, 2015.

10. Bandiera A, Bonifacio D, Manfioletti G, Mantovani F, Rustighi A, Zanconati F, Fusco A, Di Bonito L and Giancotti V: Expression of HMGI(Y) proteins in squamous intraepithelial and invasive lesions of the uterine cervix. Cancer Res 58: 426-431, 1998.

11. Fu X, Du XQ and Hao H: Study on the expression and clinical significance of high mobility group protein HMGB1 in cervical squamous cell carcinoma. Chinese Journal of Cancer Control 15: 357-359, 2008.

12. Sheng X, Du X, Zhang X, Li D, Lu C, Li Q, Ma Z, Song Q and Wang C: Clinical value of serum HMGB1 levels in early detection of recurrent squamous cell carcinoma of uterine cervix: comparison with serum SCCA, CYFRA21-1, and CEA levels. Croat Med J 50: 455-464, 2009.

13. Mitola S, Belleri M, Urbinati C, Coltrini D, Sparatore B, Pedrazzi M, Melloni E and Presta M: Cutting edge: extracellular high mobility group box-1 protein is a proangiogenic cytokine. $\mathrm{J}$ Immunol 176: 12-15, 2006.

14. Weng H, Deng Y, Xie Y, Liu H and Gong F: Expression and significance of HMGB1, TLR4 and NF- $\varkappa$ B p65 in human epidermal tumors. BMC Cancer 13: 311, 2013.

15. Wang JL, Wu DW, Cheng ZZ, Han WZ, Xu SW and Sun NN: Expression of high mobility group box-B1 (HMGB-1) and matrix metalloproteinase-9 (MMP-9) in non-small cell lung cancer (NSCLC). Asian Pac J Cancer Prev 15: 4865-4869, 2014.

16. Yin M, Xu Y, Lou G, Hou Y, Meng F, Zhang H, Li C and Zhou R: LAPTM4B overexpression is a novel predictor of epithelial ovarian carcinoma metastasis. Int J Cancer 129: 629-635, 2011.

17. Lipari MT, Li W, Moran P, Kong-Beltran M, Sai T, Lai J, Lin SJ, Kolumam G, Zavala-Solorio J, Izrael-Tomasevic A, et al: Furin-cleaved proprotein convertase subtilisin/kexin type 9 (PCSK9) is active and modulates low density lipoprotein receptor and serum cholesterol levels. J Biol Chem 287: 43482-43491, 2012.

18. Goulet B, Chan G, Chambers AF and Lewis JD: An emerging role for the nuclear localization of maspin in the suppression of tumor progression and metastasis. Biochem Cell Biol 90: 22-38, 2012.

19. Alvarez Secord A, Darcy KM, Hutson A, Huang Z, Lee PS, Jewell EL, Havrilesky LJ, Markman M, Muggia F and Murphy SK: The regulation of MASPIN expression in epithelial ovarian cancer: association with $p 53$ status, and MASPIN promoter methylation: a gynecologic oncology group study. Gynecol Oncol 123: 314-319, 2011.

20. Zhang C, Zhang M, Wu Q, Peng J, Ruan Y and Gu J: Hepsin inhibits CDK11p58 IRES activity by suppressing unr expression and eIF- $2 \alpha$ phosphorylation in prostate cancer. Cell Signal 27: 789-797, 2015. 
21. Zhang M, Zhao J, Tang W, Wang Y, Peng P, Li L, Song S, Wu H, Li C, Yang C, et al: High Hepsin expression predicts poor prognosis in gastric cancer. Sci Rep 6: 36902, 2016

22. Pelkonen M, Luostari K, Tengström M, Ahonen H, Berdel B, Kataja V, Soini Y, Kosma VM and Mannermaa A: Low expression levels of hepsin and TMPRSS3 are associated with poor breast cancer survival. BMC Cancer 15: 431, 2015.

23. Stephan C, Yousef GM, Scorilas A, Jung K, Jung M, Kristiansen G, Hauptmann S, Kishi T, Nakamura T, Loening SA, et al: Hepsin is highly overexpressed in and a new candidate for a prognostic indicator in prostate cancer. J Urol 171: 187-191, 2004.

24. Matsuo T, Nakamura K, Takamoto N, Kodama J, Hongo A, Abrzua F, Nasu Y, Kumon H and Hiramatsu Y: Expression of the serine protease hepsin and clinical outcome of human endometrial cancer. Anticancer Res 28 (1A): 159-164, 2008.
25. Lucas JM, True L, Hawley S, Matsumura M, Morrissey C, Vessella R and Nelson PS: The androgen-regulated type II serine protease TMPRSS2 is differentially expressed and mislocalized in prostate adenocarcinoma. J Pathol 215: 118-125, 2008.

26. Wilson S, Greer B, Hooper J, Zijlstra A, Walker B, Quigley J and Hawthorne S: The membrane-anchored serine protease, TMPRSS2, activates PAR-2 in prostate cancer cells. Biochem J 388: 967-972, 2005. 